RESCEU-9/97

\title{
Flattening the Inflaton's Potential with Quantum Corrections II
}

\author{
Ewan D. Stewart \\ Research Center for the Early Universe \\ University of Tokyo \\ Tokyo 113, Japan
}

October 3, 2018

\begin{abstract}
In a previous paper I showed that a classical scalar potential with $V^{\prime \prime} / V \sim 1$ can be sufficiently flattened by quantum corrections to give rise to slow-roll inflation. In this paper I give a hybrid inflation implementation of that idea which can naturally produce a spectral index in the observationally viable range even for $V^{1 / 4} \sim 10^{10}$ to $10^{11} \mathrm{GeV}$. Although any observationally viable spectral index can be obtained, the model does predict a distinctive spectral shape.
\end{abstract}




\section{Introduction}

Slow-roll inflation [1, 2] requires an unusually flat scalar potential. This is quantified by the conditions

$$
\left(\frac{V^{\prime}}{V}\right)^{2} \ll \frac{1}{M_{\mathrm{Pl}}^{2}}
$$

and

$$
\left|\frac{V^{\prime \prime}}{V}\right| \ll \frac{1}{M_{\mathrm{Pl}}^{2}}
$$

where $M_{\mathrm{Pl}}=1 / \sqrt{8 \pi G}$. The first condition is generally not difficult to achieve; one simply needs to be sufficiently near an extremum of the potential with positive potential energy. In many cases 'sufficiently' just means $\ll M_{\mathrm{Pl}}$ from the extremum, which is almost difficult to avoid!

However, the second condition presents the most, perhaps only, serious obstacle to building a model of inflation [3, 4]. This is because the positive potential energy required for inflation spontaneously breaks supersymmetry', generically inducing

$$
\left|\frac{V^{\prime \prime}}{V}\right| \gtrsim \frac{1}{M_{\mathrm{Pl}}^{2}}
$$

for all scalar fields [6, 3, 田, and in particular for the inflaton [3, 4]. The $\sim$ holds when the field has no supersymmetric mass and the supersymmetry breaking is only communicated via gravitational strength interactions.

Many models of inflation are built ignoring gravitational strength interactions, and so are implicitly setting $M_{\mathrm{Pl}}=\infty$. Clearly one cannot achieve Eq. (2) in this context. Models of inflation built in the context of supergravity have traditionally resolved this problem by fine tuning, either explicit or implicit. Only recently have there been any plausible proposals for solving this problem.

The first was given in Refs. [3, 4]. It employed forms for the Kahler potential that had been derived from weakly coupled heterotic string theory, in combination with a subset of the modular symmetries, to cancel the supergravity corrections. This method should also work in appropriate limits of M-theory. It requires an inflationary energy density well above the vacuum supersymmetry breaking scale, $V^{1 / 4} \gg M_{\mathrm{s}} ; M_{\mathrm{s}} \sim 10^{10}$ to $10^{11} \mathrm{GeV}$ in our vacuum for gravity mediated supersymmetry breaking. A natural implementation of this method would be to have the non-perturbative physics that leads to gaugino condensation at a scale

\footnotetext{
${ }^{1}$ To build a model of inflation, not to mention the rest of particle physics, in the absence of supersymmetry seems hopeless. For an introduction to supersymmetry and supergravity, see Ref. 河.

2 Natural inflation [7] naturally achieves a small $V^{\prime \prime}$ by assuming an approximate global $U(1)$ symmetry, but does not naturally satisfy Eq. (2) because $V$ vanishes in the limit where the symmetry is exact. A hybrid natural inflation model might avoid this problem though, as was noted in Ref. [8].
} 
$\Lambda_{\mathrm{gc}} \sim 10^{13} \mathrm{GeV}$ in our vacuum generate the inflationary potential at a similar energy scale $V^{1 / 4} \sim \Lambda_{\mathrm{gc}}$. It should be possible to stabilise the moduli during inflation by the same method that stabilises them in our vacuum, though the transition from inflation to vacuum could be dangerous if the end of inflation is not sufficiently smooth. A simple model of inflation with the appropriate energy scale and a smooth end to inflation was given in Ref. 9]. This method has the practical disadvantage that it requires detailed control of the effective supergravity theory, but otherwise remains very promising.

It was also noted in Ref. [四] that the supergravity corrections could be avoided if the inflationary potential energy was dominated by the $D$-term, and a hybrid inflation [10, 3] implementation of that idea was constructed with a Fayet-Iliopoulos term dominating the energy density. The main problem with this method is to obtain a Fayet-Iliopoulos term at a low enough energy scale to obtain the COBE normalisation for the density perturbations, but at a scale higher than the $F$-term supersymmetry breaking to avoid the $F$-term supersymmetry breaking inducing too large a mass for the inflaton. The stabilisation of the moduli, the dilaton in the case of the heterotic string, may also be a serious problem. A FayetIliopoulos term does arise in many compactifications of the heterotic string [11], but its scale, typically $V_{\mathrm{FI}}^{1 / 4} \sim 10^{17}$ to $10^{18} \mathrm{GeV}$, a conservative lower bound being $V_{\mathrm{FI}}^{1 / 4}>2 \times 10^{16} \mathrm{GeV}$, is at the limit of being too high for any type of inflation to meet the COBE constraint [4], and in particular is always too high if the slope of the inflaton's potential is dominated by the loop correction as in Ref. [12], in which case the COBE normalisation requires $V_{\text {inf }}^{1 / 4} \sim 5 \times 10^{15} \mathrm{GeV}$. However, in M-theory scales are more flexible [13], and so it may be possible to obtain a Fayet-Iliopoulos term at a sufficiently low scale. There still remains the problem of stabilising the moduli. One possibility would be if either the gauge coupling, or the Fayet-Iliopoulos term itself, had a large non-perturbative dependence on the moduli. Whether this can be achieved at the same time as having a sufficiently low energy scale for the Fayet-Iliopoulos term remains to be seen. Another possibility would be to generate the Fayet-Iliopoulos term by field theory methods at a lower energy scale, but as this probably requires $F$-term supersymmetry breaking it may be difficult to avoid the inflaton getting too large a mass from this source. Especially in this latter case, one might also have to consider the inflaton dependence of the gauge coupling and Fayet-Iliopoulos term, which could provide an effective mass for the inflaton.

The method of [14] uses a global Heisenberg symmetry, which has been derived from string theory at tree and one loop level, to cancel the supergravity corrections. The stabilisation of the modulus that forms an integral part of the mechanism is a serious problem for this method. This is the only method that could conceivably implement the naively popular $\phi^{n}$ chaotic inflation models.

The method of [15] is somewhat complex and requires specific couplings of a Goldstone boson to the inflaton, but is an interesting possibility. 
In this paper I will use the method of Ref. 8]. It has the advantages that it does not require any special features of the high energy theory, and can be implemented at the scale $V^{1 / 4} \sim 10^{10}$ to $10^{11} \mathrm{GeV}$ where the moduli are already stabilised.

I will rely heavily on Ref. [8]. The reader would benefit from reading it first. From now on I set $M_{\mathrm{Pl}}=1$. The potential of the model (see Figure 1) is [8]

$$
V(\phi)=V_{0}\left[1-\frac{1}{2} f(\epsilon \ln \phi) \phi^{2}+\ldots\right]
$$

with $f(0) \sim 1$, the minimum value expected in a generic supergravity theory, and $\epsilon \ll 1$. The function $f$ is determined by the renormalisation group running of the mass of the inflaton $\phi$. The potential is assumed to have a maximum at $\phi=\phi_{*}$, with $V_{0}^{1 / 2} \ll \phi_{*} \ll 1$. We then have $f_{*}=\mathcal{O}(\epsilon)$, allowing slow roll inflation near $\phi_{*}$ [8]. We are free to choose $\phi_{*}=e^{-1 / \epsilon}$, so that $f_{*}=f(-1)$.

The most natural scale for the potential is $V_{0} \sim M_{\mathrm{s}}^{4}$, where $M_{\mathrm{s}}$ is the supersymmetry breaking scale in our vacuum. I will take $M_{\mathrm{s}} \sim 10^{-8}$ corresponding to gravity mediated supersymmetry breaking. In this case the COBE normalisation gives $\phi_{*} \sim 10^{-11}$ and so $\epsilon \simeq 0.04$ [8]. In Section 2, $\epsilon$ will be derived from a gauge coupling of strength similar to that of the GUT gauge coupling inferred from the LEP data, $\alpha_{\mathrm{GUT}} \sim 0.04$. It is worth noting that the fact that $M_{\mathrm{S}}$ is so small is crucial to our being able to achieve slow roll inflation without fine tuning.

\section{The renormalisation group}

In this section I will give a rough derivation of the function $f(\epsilon \ln \phi)$. The inflaton $\phi$ gives masses proportional to its expectation value to the fields to which it couples, and so $\phi$ acts like an infra-red cutoff to the renormalisation. I will assume that $\phi$ is charged under some asymptotically free gauge group and for simplicity neglect the Yukawa couplings. Defining

$$
x \equiv \epsilon \ln \phi
$$

so that $x_{*}=-1$, the renormalisation group equation [5] for $\phi$ 's mass becomes

$$
\frac{d m_{\phi}^{2}}{d x}=-\frac{2 c \alpha}{\pi \epsilon} \tilde{m}^{2}
$$

where $c$ is the quadratic Casimir invariant of $\phi$ 's representation under the gauge group. For example, $c=3 / 4$ for a fundamental representation of $\mathrm{SU}(2)$ and $c=4 / 3$ for that of $\mathrm{SU}(3) . \alpha=g_{\text {gauge }}^{2} / 4 \pi$, where $g_{\text {gauge }}$ is the gauge coupling. $\tilde{m}$ is the gaugino mass. The renormalisation of $\alpha$ is given by

$$
\frac{d \alpha}{d x}=-\frac{b \alpha^{2}}{2 \pi \epsilon}
$$


where, for example, $b=3 N_{\mathrm{c}}-N_{\mathrm{f}}$ for an $\mathrm{SU}\left(N_{\mathrm{c}}\right)$ gauge group with $N_{\mathrm{f}}$ pairs of fundamentals and antifundamentals. $b>0$ corresponds to an asymptotically free gauge group. The renormalisation of $\tilde{m}$ is given by

$$
\tilde{m}(x)=\frac{\alpha(x) \tilde{m}(0)}{\alpha(0)} .
$$

Integrating these equations gives

$$
m_{\phi}^{2}(x)=-m_{\phi}^{2}(0)\left[A\left(\frac{1}{1+\frac{b}{2 \pi} \frac{\alpha(0)}{\epsilon} x}\right)^{2}-(A+1)\right]
$$

where

$$
A=\frac{2 c}{b}\left(\frac{\tilde{m}^{2}(0)}{-m_{\phi}^{2}(0)}\right) .
$$

To lowest order, $\epsilon$ is defined by $m_{\phi}^{2}(-1)=0$, which gives

$$
\epsilon=\alpha(0) \frac{b}{2 \pi}[A+1+\sqrt{A(A+1)}] .
$$

Therefore

$$
m_{\phi}^{2}(x)=-(A+1) m_{\phi}^{2}(0)\left[\left(\frac{y_{\infty}}{y_{\infty}+1+x}\right)^{2}-1\right]
$$

where

$$
y_{\infty}=A+\sqrt{A(A+1)} .
$$

The potential, Eq. (画), is then

$$
V(\phi)=V_{0}+\frac{1}{2} m_{\phi}^{2}(\epsilon \ln \phi) \phi^{2}+\ldots
$$

with $m_{\phi}^{2}(\epsilon \ln \phi)$ given by Eq. (12) and $m_{\phi}^{2}(0) \sim-V_{0}$.

\section{Initial conditions}

In Ref. [8], I said that the above model with the slow roll inflation occuring as $\phi$ rolls from the maximum at $\phi=\phi_{*}$ towards the false vacuum at $\phi=0$, had problematic initial conditions. Here, I argue to the contrary that this model has very natural initial conditions if we choose the vacuum at $\phi>\phi_{*}$ to be another false vacuum. Then the inflaton $\phi$ will be easily trapped in the large false vacuum potential well at $\phi>\phi_{*}$, giving rise to eternal [2] old inflation. There will be an extremely small, but finite, chance that quantum fluctuations will kick $\phi$ to the top of the barrier at $\phi=\phi_{*}$, where once again eternal inflation occurs. The chance that this will happen is extremely small, but, because it is finite, it will be more than compensated for by the two lots of eternal inflation. Thus the initial conditions for the slow roll inflation, $\phi=\phi_{*}$, are naturally obtained. 


\section{Slow roll inflation}

As we are focussing on the case where the slow roll inflation occurs as $\phi$ rolls off the maximum at $\phi=\phi_{*}$ towards the false vacuum at $\phi=0$, it will be convenient to define

$$
y \equiv \epsilon \ln \left(\frac{\phi_{*}}{\phi}\right)=-1-x,
$$

so that $y_{*}=0$ and $y>0$ during the slow roll inflation, and

$$
g(y) \equiv-f(x)-\frac{\epsilon}{2} f^{\prime}(x)
$$

so that $g_{*}=0, g>0$ during the slow roll inflation, and $g_{*}^{\prime}=f_{*}^{\prime}+\mathcal{O}(\epsilon)$. Eq. (19) of Ref. [8] then becomes

$$
\tau=\epsilon H t=\frac{2}{3} \int \frac{d y}{1-\sqrt{1-4 g / 3}} .
$$

Following Section 2, we take as an example

$$
g(y)=a\left[\left(\frac{y_{\infty}}{y_{\infty}-y}\right)^{2}-1\right]
$$

with

$$
a=(1+A) f(0)+\mathcal{O}(\epsilon)
$$

and $y_{\infty}$ given by Eq. (13). Then

$$
g_{*}^{\prime}=\frac{2 a}{y_{\infty}} .
$$

With this choice for $g$, we can integrate Eq. (17) to give

$\tau=\frac{1}{g_{*}^{\prime}}\left\{\ln y+\left(1-\frac{y}{y_{\infty}}\right)\left(1+\sqrt{1-\frac{4 g}{3}}\right)-\ln \left[1+\left(1-\frac{y}{y_{\infty}}\right) \sqrt{1-\frac{4 g}{3}}\right]-2+\ln 2\right\}$.

This is only valid for $g<g_{\mathrm{fr}}=3 / 4$ or

$$
y<y_{\text {fr }}=y_{\infty}\left(1-\frac{1}{\sqrt{1+\frac{3}{4 a}}}\right)
$$

or

$$
\tau<\tau_{\text {fr }}=\frac{1}{g_{*}^{\prime}}\left(\ln y_{\text {fr }}+\frac{1}{\sqrt{1+\frac{3}{4 a}}}-2+\ln 2\right) .
$$

For $\tau>\tau_{\text {fr }}, \phi$ fast rolls towards $\phi=0$. I will neglect the small number of $e$-folds of inflation during this fast rolling stage. During slow roll, $y \ll 1$ and $g \ll 1$, and so 8

$$
\tau \simeq \tau_{\mathrm{sr}}=\frac{1}{g_{*}^{\prime}} \ln y
$$




\section{$5 \quad$ Ending inflation}

To end inflation, one must use a hybrid inflation type mechanism [10, 9, 16] to exit from the false vacuum at some critical value $\phi_{\mathrm{c}}<\phi_{*}$.

A standard hybrid inflation exit from the false vacuum [10], with the new field $\psi$ also having a mass squared $m_{\psi}^{2} \sim V_{0}$ [17], and with a coupling $\lambda \lesssim 1$ between the fields,

$$
V(\phi, \psi)=V_{0}+\frac{1}{2}[g(y)+\mathcal{O}(\epsilon)] V_{0} \phi^{2}-\frac{1}{2} m_{\psi}^{2} \psi^{2}+\frac{1}{2} \lambda^{2} \phi^{2} \psi^{2}+\ldots
$$

would give $\phi_{\mathrm{c}}=m_{\psi} / \lambda \gtrsim 10^{-16}$, or $y_{\mathrm{c}} \lesssim 0.46$. Typically, $g_{\mathrm{c}}>3 / 4$, in which case $\phi$ will be fast rolling at $\phi=\phi_{\mathrm{c}}$ making the precise value of $\phi_{\mathrm{c}}$ not very important.

Such a hybrid inflation exit from the false vacuum may lead to a large spike in the spectrum [17], which could be dangerous. In our model, contrary to that of Ref. [17], the inflaton is typically fast rolling at $\phi=\phi_{\mathrm{c}}$ which may make the spike less dangerous [17]. If dangerous, such a spike could be avoided by a mutated hybrid inflation type exit from the false vacuum [9, 18, 16]. For example, one could naturally have an additional term $\sim V_{0} \phi \psi$ in the potential which would avoid the spike but leave $\phi_{\mathrm{c}}$ essentially unchanged. As the gauge coupling is getting stronger as $\phi$ is decreasing, it is also conceivable that the exit from the false vacuum might be controlled by strong coupling effects.

Finally, there will be a stage of non-slow roll inflation [17] in which $\psi$ rolls from $\psi=\psi_{\text {init }} \sim 10^{-16}$ to our vacuum at $\psi=\psi_{\text {vac }} \sim 1$. It will last for

$$
\begin{aligned}
N_{\psi} & =\frac{V_{0}}{2 m_{\psi}^{2}}\left(1+\sqrt{1+\frac{4 m_{\psi}^{2}}{3 V_{0}}}\right) \ln \frac{\psi_{\text {vac }}}{\psi_{\text {init }}} \\
& \sim \frac{37 V_{0}}{2 m_{\psi}^{2}}\left(1+\sqrt{1+\frac{4 m_{\psi}^{2}}{3 V_{0}}}\right)
\end{aligned}
$$

$e$-folds.

For $V_{0} \sim 10^{-32}$, and assuming that thermal inflation 19 occurs after this inflation, observable scales would leave the horizon between 20 and $30 e$-folds before the end of this inflation [20]. If reheating was rapid and there was no thermal inflation, this would be increased to between 40 and $50 e$-folds at maximum. For $m_{\psi}^{2}=V_{0}$, Eq. (27) gives $N_{\psi}=47$, so that even in the latter case observable scales would leave the horizon during this non-slow roll stage of inflation leading to an unacceptable spectral index. To get observable scales safely leaving the horizon during the slow roll inflation, say at least between 10 and $20 e$-folds before $\phi$ reached $\phi=\phi_{\mathrm{c}}$, would require $N_{\psi} \lesssim 10$ and so $m_{\psi}^{2} \gtrsim 8 V_{0}$ in the case with thermal inflation, and $N_{\psi} \lesssim 30$ and so $m_{\psi}^{2} \gtrsim 1.7 V_{0}$ in the case without. The latter requirement is clearly not very severe, and even the former is not unreasonable. For example, $V(\psi)=\frac{1}{2} V_{0}[1+\cos (2 \pi \psi)]$, which has a period of one, gives 
$m_{\psi}^{2}=2 \pi^{2} V_{0}$. However, I do not find this feature of the model entirely satisfactory in the case with thermal inflation.

\section{The spectral index}

To lowest order in the slow roll approximation, the spectral index is [8]

$$
n=1+2 \frac{V^{\prime \prime}}{V}=1+2 g_{*}^{\prime}(y-\epsilon) .
$$

Eqs. (20), (22), (23) and (24) then give

$$
n=1-2 \epsilon g_{*}^{\prime}+8 a\left(1-\frac{1}{\sqrt{1+\frac{3}{4 a}}}\right) \exp \left[-\epsilon g_{*}^{\prime}\left(N-N_{\mathrm{fr}}\right)-2+\frac{1}{\sqrt{1+\frac{3}{4 a}}}\right]
$$

where $N-N_{\text {fr }}=\left(\tau_{\text {fr }}-\tau\right) / \epsilon$ is the number of $e$-folds of inflation from the scale $k \propto$ $e^{-N}$ leaving the horizon to $\phi=\phi_{\mathrm{fr}}$. From the discussion of Section 5, one might expect observable scales to leave the horizon during the interval $N-N_{\text {fr }} \sim 10$ to 20. I have been assuming that $\phi_{\mathrm{c}}<\phi_{\mathrm{fr}}$, as will usually be the case. If $\phi_{\mathrm{c}}>\phi_{\mathrm{fr}}$, inflation may end slightly earlier so that one should take a slightly larger value of $N-N_{\text {fr. }}$.

A more accurate but implicit formula is given by using [21

$$
\begin{aligned}
n & =1+2 \frac{V^{\prime \prime}}{V}+2.13 \frac{V^{\prime} V^{\prime \prime \prime}}{V^{2}}+\frac{2}{3}\left(\frac{V^{\prime \prime}}{V}\right)^{2} \\
& =1+2\left(g-\epsilon g^{\prime}\right)-2.13 \epsilon g\left(g^{\prime}-\epsilon g^{\prime \prime}\right)+\frac{2}{3}\left(g-\epsilon g^{\prime}\right)^{2}
\end{aligned}
$$

and Eq. (21) instead of Eq. (24). Some example spectra and spectral indices are plotted in Figures 2 and 3 respectively.

\section{Conclusions}

In a previous paper [8] I described how to construct a potential flat enough for slow roll inflation without fine tuning and without making any assumptions about the high energy theory. In this paper I have emphasised an implementation of that idea which can naturally produce an observationally viable spectral index even for $V^{1 / 4} \sim 10^{10}$ to $10^{11} \mathrm{GeV}$. Although any observationally viable spectral index can be obtained, the model does predict a distinctive shape to the spectrum, given to lowest order in the slow roll approximation by

$$
P(k)=Q k^{-2 \nu} \exp \left(\sigma k^{\nu}\right)
$$


or in terms of the spectral index

$$
n(k)=1-2 \nu+\sigma \nu k^{\nu}
$$

which may allow it to be distinguished from other models of inflation by sufficiently precise observations. The extra power on small scales, for a given tilt on larger scales, may be helpful for mixed dark matter scenarios of structure formation.

Finally, I will compare the model with that of Randall, Soljacic and Guth (RSG) [17]. The models share some of the same motivations and features, such as to use the flat directions characteristic of supersymmetric and string theories, lifted by supersymmetry breaking at a scale $M_{\mathrm{s}} \sim 10^{10}$ to $10^{11} \mathrm{GeV}$, to drive inflation. Both also use a hybrid inflation type mechanism to exit from the false vacuum. However, they also have important differences:

1. RSG fine tune the inflaton's mass while our model uses the method of Ref. [8], i.e. the renormalisation of the inflaton's mass that would be present anyway, to obtain a mass small enough for slow roll inflation without fine tuning.

2. The numbers tend to work out better here, e.g. the coupling $\lambda$ involved in the hybrid inflation mechanism does not need to be small; our small parameter $\epsilon$ is derived from a gauge coupling of the order of the GUT gauge coupling inferred from the LEP data. The only parameter we may have to tune slightly is the mass of the other field involved in the hybrid inflation mechanism, but even this was to a lesser extent than in RSG.

3. The possible spike in the spectrum produced by the hybrid inflation mechanism is probably less dangerous here because the inflaton will typically be fast rolling at this point.

4. The spectral index in RSG is $n>1$, while our model can give $n<1$ which may be preferred by observations.

5. The spectrum has a significant bend. It is not clear whether this is an advantage or not, but is at least observationally interesting.

\section{Acknowledgements}

I thank David Lyth for helpful discussions. I am supported by a JSPS Fellowship at RESCEU, and my work is supported by Monbusho Grant-in-Aid for JSPS Fellows No. 96184. 


\section{References}

[1] E. W. Kolb and M. S. Turner, The Early Universe (Addison-Wesley, New York, 1990).

[2] A. D. Linde, Particle Physics and Inflationary Cosmology (Harwood Academic, Switzerland, 1990).

[3] E. J. Copeland, A. R. Liddle, D. H. Lyth, E. D. Stewart and D. Wands, astro-ph/9401011, Phys. Rev. D 49, 6410 (1994).

[4] E. D. Stewart, hep-ph/9405389, Phys. Rev. D 51, 6847 (1995).

[5] H. P. Nilles, Phys. Rep. 110, 1 (1984); D. Bailin and A. Love, Supersymmetric Gauge Field Theory and String Theory (IOP, Bristol, 1994).

[6] M. Dine, W. Fischler and D. Nemeschansky, Phys. Lett. B 136, 169 (1984).

[7] K. Freese, J. A. Frieman and A. V. Olinto, Phys. Rev. Lett. 65, 3233 (1990); F. C. Adams, J. R. Bond, K. Freese, J. A. Frieman and A. V. Olinto, hepph/9207245, Phys. Rev. D 47, 426 (1993).

[8] E. D. Stewart, hep-ph/9606241, Phys. Lett. B 391, 34 (1997).

[9] E. D. Stewart, astro-ph/9407040, Phys. Lett. B 345, 414 (1995).

[10] A. D. Linde, Phys. Lett. B 259, 38 (1991).

[11] M. Dine, N. Seiberg and E. Witten, Nucl. Phys. B 289, 589 (1987).

[12] P. Binetruy and G. Dvali, hep-ph/9606342, Phys. Lett. B 388, 241 (1996); E. Halyo, hep-ph/9606423, Phys. Lett. B 387, 43 (1996).

[13] E. Witten, hep-th/9602070, Nucl. Phys. B 471, 135 (1996).

[14] M. K. Gaillard, H. Murayama and K. A. Olive, hep-ph/9504307, Phys. Lett. B 355, 71 (1995).

[15] J. A. Adams, G. G. Ross and S. Sarkar, hep-ph/9608336, Phys. Lett. B 391, 271 (1997).

[16] D. H. Lyth and E. D. Stewart, hep-ph/9606412, Phys. Rev. D 54, 7186 (1996).

[17] L. Randall, M. Soljacic and A. H. Guth, hep-ph/9512439, Nucl. Phys. B 472, 377 (1996); hep-ph/9601296.

[18] J. Garcia-Bellido, A. D. Linde and D. Wands, astro-ph/9605094, Phys. Rev. D 54, 6040 (1996). 
[19] D. H. Lyth and E. D. Stewart, hep-ph/9510204, Phys. Rev. D 53, 1784 (1996).

[20] A. R. Liddle and D. H. Lyth, astro-ph/9303019, Phys. Rep. 231, 1 (1993).

[21] E. D. Stewart and D. H. Lyth, gr-qc/9302019, Phys. Lett. B 302, 171 (1993). 


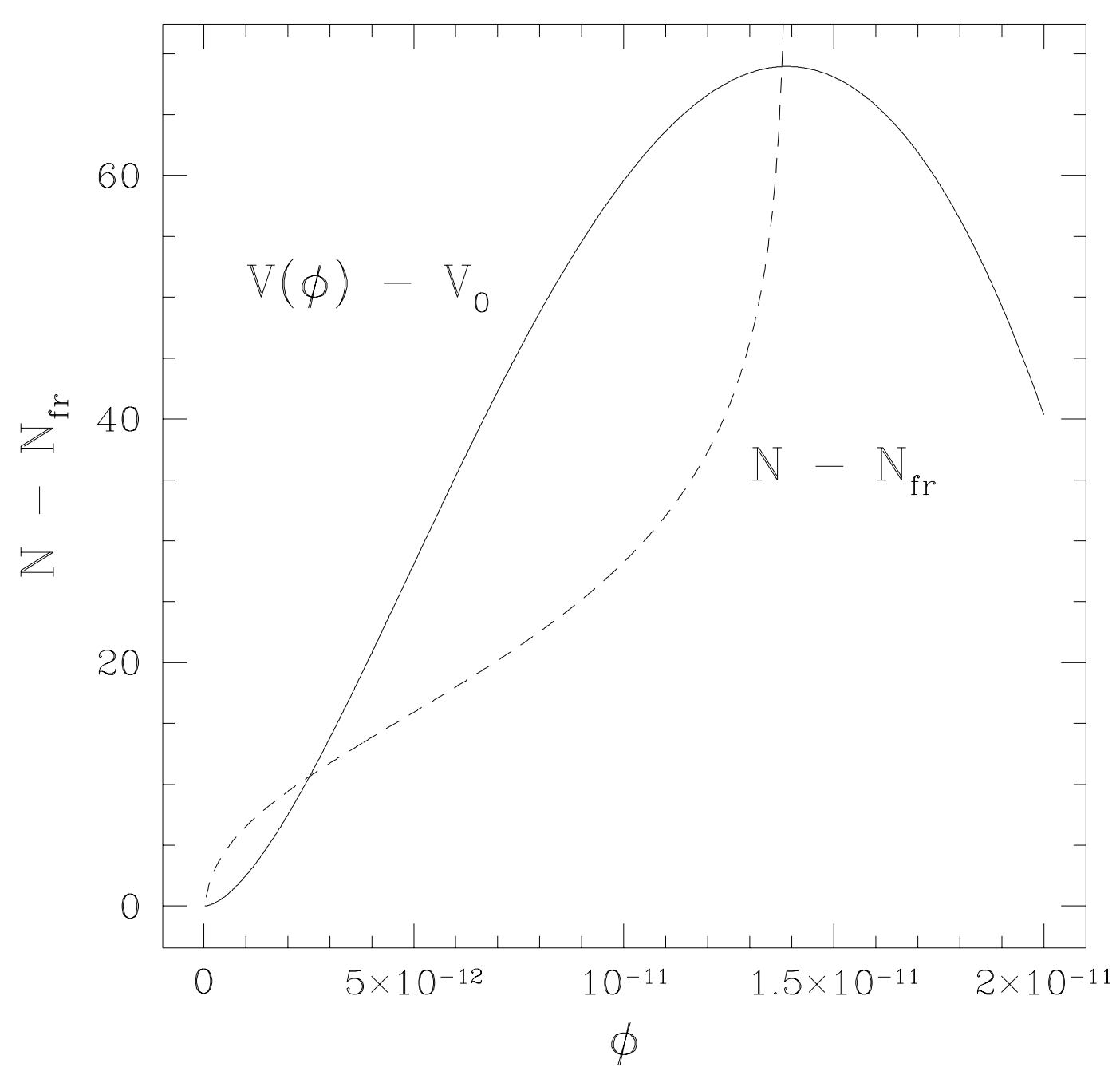

Figure 1: The potential Eq. (4), with $f$ given by Eqs. (16) and (18), for $f(0)=1$ and $A=0.5$. The potential energy at $\phi=0$ is subtracted and the normalisation is arbitrary. The number of $e$-folds of inflation until the inflaton starts to fast roll towards $\phi=0$ is plotted on the same graph. The smallest and largest observable scales might be expected to correspond to $N-N_{\text {fr }} \sim 10$ and 20, respectively, though there is considerable uncertainty in this estimate. 


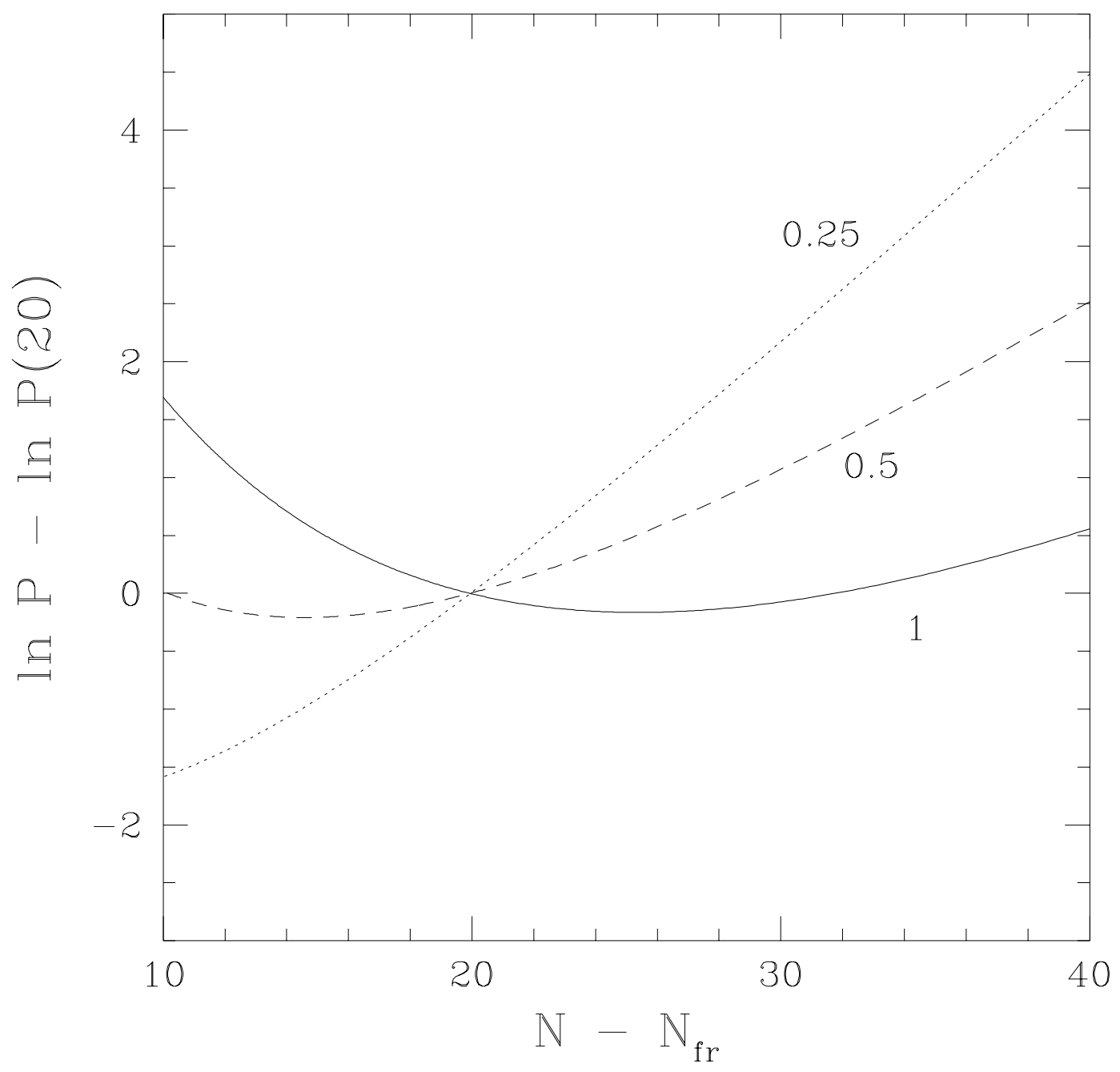

Figure 2: The spectra for $f(0)=1$ and the displayed values of $A$. 


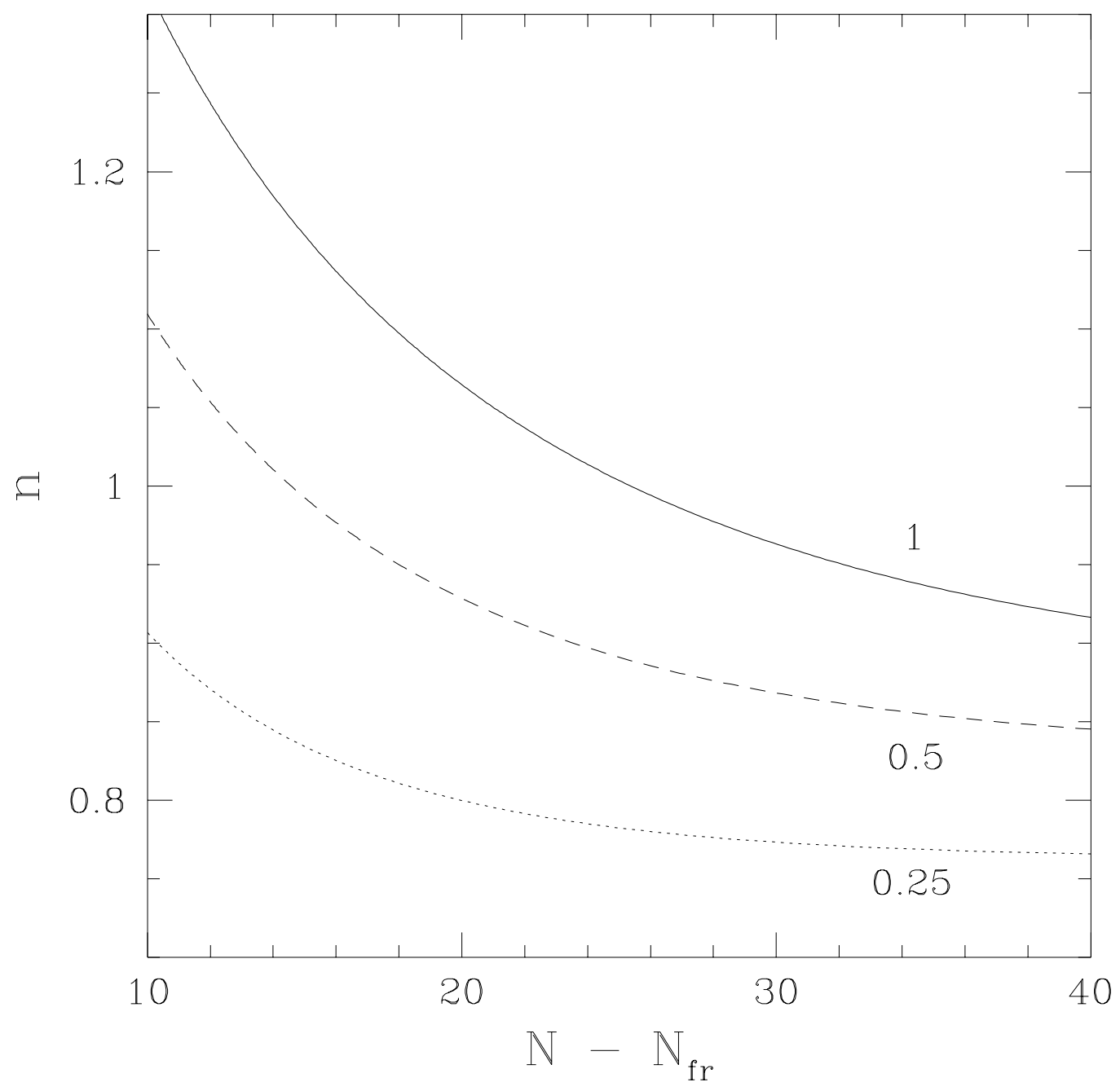

Figure 3: The spectral indices for $f(0)=1$ and the displayed values of $A$. 